\title{
The Importance of Partial Voluming in Multi-dimensional Medical Image Segmentation
}

\author{
Maja Pokrić, Neil Thacker, Marietta L.J. Scott, and Alan Jackson \\ Imaging Science and Biomedical Engineering \\ School of Medicine \\ University of Manchester \\ Manchester M13 9PT, UK \\ maja.pokric@man.ac.uk
}

\begin{abstract}
The presented method addresses the problem of multi-spectral image segmentation through use of a model which takes into account partial volumes of tissues being present in a single voxel at boundaries. The parameters of the multi-dimensional model of pure tissues and their mixtures are iteratively adjusted using an Expectation Maximisation (EM) optimisation technique. Bayes theory is used to generate probability maps for each segmented tissue which estimates the most likely tissue volume fraction within each voxel.
\end{abstract}

A common approach for medical image segmentation involves modelling only pure tissue intensity distributions. However, the accurate interpretation of the data requires that partial volume distributions for a mixture of tissues that can be present in any given voxel must be modelled [1. Knowledge of the physics of image formation in a wide variety of medical image data allows that partial volume distributions for paired tissue combinations can be modelled as a linear process. Hence, we adopted approach whereby the pure tissues have been modelled using a multi-dimensional Gaussian distribution, while mixtures of tissues take the novel form of a triangular distribution convolved with a Gaussian. A multi-variate Gaussian distribution for multi-dimensional data $\mathbf{g}$ for each pure tissue $t$ is defined as:

$$
d_{t}(\mathbf{g})=\alpha_{t} e^{-\left(\mathbf{g}-\mathbf{M}_{\mathbf{t}}\right)^{T} C_{t}\left(\mathbf{g}-\mathbf{M}_{\mathbf{t}}\right)}
$$

where: (i) $\mathbf{M}_{\mathbf{t}}$ is a mean tissue vector; (ii) $C_{t}$ is an inverse of covariance matrix; (iii) $\alpha_{t}$ is a constant which gives unit normalisation. Multi-dimensional partial volume distributions is modelled along the line between two pure tissue means $\mathbf{M}_{\mathbf{t}}$ and $\mathbf{M}_{\mathbf{s}}$ :

$$
d_{t s}(\mathbf{g})=\beta_{t s} T_{t s}(h) e^{-(\mathbf{g}-\mathbf{h} \cdot \mathbf{g} /|\mathbf{h}|)^{T} C_{h}(\mathbf{g}-\mathbf{h} \cdot \mathbf{g} /|h|)}
$$

where: (i) $h$ is a fractional distance between two centres of distribution $[0<h<$ $1], h=\left(\mathbf{g}-\mathbf{M}_{\mathbf{s}}\right) C_{h}\left(\mathbf{M}_{\mathbf{t}}-\mathbf{M}_{\mathbf{s}}\right) /\left|\left(\mathbf{M}_{\mathbf{t}}-\mathbf{M}_{\mathbf{s}}\right) C_{h}\left(\mathbf{M}_{\mathbf{t}}-\mathbf{M}_{\mathbf{s}}\right)\right|$; (ii) $T_{t s}(h)$ is the $1 \mathrm{D}$ partial volume distribution for tissue $t$ generated by partial voluming process 
with tissue $s$ (iii) $C_{h}$ is an inverse covariance matrix, $C_{h}=C_{t} h+C_{s}(1-h)$; (iv) $\beta_{t s}$ is a constant which gives unit normalisation.

Parameters of the model can be iteratively estimated using the Expectation Maximisation (EM) approach 23. EM is used to estimate the parameters by maximising the likelihood of the data distribution. This involves first getting from the likelihood distributions defined above to a probability of a given tissue proportion given the data $P\left(t \mid \mathbf{g}_{\mathbf{v}}\right)$. The conditional probability of a grey level being due to a certain mechanism $n$ (either a pure or mixture tissue component) can be calculated using Bayes theory, as follows:

$$
P(n \mid \mathbf{g})=\frac{d_{n}(\mathbf{g}) f_{n}}{f_{0}+\sum_{t} d_{t}(\mathbf{g}) f_{t}+\sum_{t} \sum_{s} d_{t s}(\mathbf{g}) f_{t s}}
$$

where $f_{n}, f_{0}, f_{t}$ and $f_{t s}$ are effectively "priors", expressed here as frequencies (i.e. number of voxels) which belong to a particular tissue type, pure tissues or partial volumes. Unknown tissues are accounted for in the Bayesian formulation by including a fixed extra term $f_{o}$ for infrequently occuring outlier data in total probability which enables separation of pathological tissues. The EM approach has been applied for the estimation of model parameters for pure and mixture tissues:

$$
\begin{aligned}
& f_{t}^{\prime}=\sum_{v}^{V} P\left(t \mid \mathbf{g}_{v}\right) \quad \text { and } \quad f_{t s}^{\prime}=f_{s t}^{\prime}=\frac{1}{2} \sum_{v}^{V} P\left(t s \mid \mathbf{g}_{v}\right)+P\left(s t \mid \mathbf{g}_{v}\right) \\
& M_{t}^{\prime}=\frac{1}{V} \sum_{v}^{V} P\left(t \mid \mathbf{g}_{v}\right) \mathbf{g}_{v} \quad \text { and } \quad C_{t}^{\prime-1}=\frac{1}{V} \sum_{v}^{V} P\left(t \mid \mathbf{g}_{v}\right)\left(\mathbf{g}_{v}-\mathbf{G}_{\mathbf{t}}\right) \otimes\left(\mathbf{g}_{v}-\mathbf{G}_{\mathbf{t}}\right)^{T}
\end{aligned}
$$

The E-step recalculates multi-dimensional probability densities, while the $\mathbf{M}$ step involves re-estimation of model parameters. Multi-spectral segmentation was performed for six different tissue classes on four co-registred MRI images by applying E- and M-steps in turns. It has been found that the algorithm converges within 10 iterations and that a partial volume model accounts for almost half of data present. This highlighs the importance of inclusion of that part of a model. Inclusion of a partial volume model leads to better visual appearance of segmented tissues as voxels are not simply classified as a single tissue, which causes artifacts at tissue boundaries. This in turn enables creation of better geometric models to be used in simulation and visualisation. This method can be applied on any sequence of images for which the linearity assumption holds.

\section{References}

1. Laidlaw, D.H., Fleischer, K.W., Barr, A.H.: Partial-volume Bayesian Classification of Material Mixtures in MR Volume Data Using Voxel Histograms. IEEE Trans. Med. Imag. 17(1) (1998) 74-86

2. Dempster, A.P.,Laird, N.M., Rubin, D.B.: Maximum Likelihood from Incomplete Data via EM Algorithm. Journal of the Royal Society 39 (1977) 1-38

3. Guillemaud, R., Brady, J.M.: Estimating the bias Field of MR Images. IEEE TRANS. on Medical Imaging 16(3) (1997) 238-251 